TIPA. Travaux interdisciplinaires sur la parole et le langage

34 | 2018

La langue des signes, c'est comme ça

\title{
Editorial : Le flambeau de la langue des signes
}

\section{Mélanie Hamm}

\section{(2) OpenEdition}

Journals

Édition électronique

URL : http://journals.openedition.org/tipa/2739

DOI : 10.4000/tipa.2739

ISSN : 2264-7082

Éditeur

Laboratoire Parole et Langage

Référence électronique

Mélanie Hamm, «Editorial : Le flambeau de la langue des signes », TIPA. Travaux interdisciplinaires sur la parole et le langage [En ligne], 34 | 2018, mis en ligne le 15 novembre 2018, consulté le 09 octobre 2020. URL : http://journals.openedition.org/tipa/2739; DOI : https://doi.org/10.4000/tipa.2739

Ce document a été généré automatiquement le 9 octobre 2020.

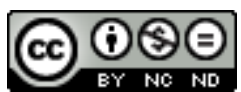

La revue TIPA. Travaux interdisciplinaires sur la parole et le langage est mise à disposition selon les termes de la licence Creative Commons Attribution - Pas d'Utilisation Commerciale - Pas de Modification 4.0 International. 


\title{
Editorial : Le flambeau de la langue des signes
}

\author{
Mélanie Hamm
}
Ce média ne peut être affiché ici. Veuillez vous reporter à l'édition en ligne http:// journals.openedition.org/tipa/2739

2 Pantomime, mimodrame, langage d'action ${ }^{1}$, gestes, expressions corporelles, mais aussi langage naturel des sourds-muets ${ }^{2}$, puis langage des sourds-muets ${ }^{3}$, langage des sourds ${ }^{4}$, mimologie ou langage mimique des sourds-muets ${ }^{5}$, français signé, français manuel, signes des mots, alphabet manuel... autant de termes qui qualifient la représentation sociale et culturelle de cette langue. Ces appellations ne sont pas sans rappeler certaines des questions que soulèvent les différentes études dans ce domaine. Mais qu'est-ce que la langue des signes? Comment l'étudier? Qu'en est-il de la linguistique des langues des signes ? Que raconte l'histoire à ce sujet?

De 1774 jusqu'aux années 1990, les travaux autour de la langue des signes portaient surtout sur les méthodes requises pour apprendre le français par la langue des signes, puis sur sa qualité de langue à part entière. Du 16ème au 18ème siècle, les pionniers de l'éducation des sourds sont - à l'exception d'Étienne de Fay (1670-1750), dit le « Vieux sourd d'Amiens" - entendants et précepteurs d'enfants de familles riches. Leur enseignement, très individualisé, repose surtout sur l'éducation de la parole. À partir de 1760, l'abbé de l'Épée (1712-1789) entreprend à son domicile parisien, l'éducation des enfants sourds-muets, en utilisant un vaste système de signes destiné à traduire fidèlement en gestes chaque mot français, en suivant au pied de la lettre la construction de la phrase ${ }^{6}$. Il les appelle : «signes méthodiques » et écrit l'Institution des sourds-muets par la voie des signes méthodiques (1776). Plus tard, Auguste Bébian (1789-1839) - élevé parmi les sourds-muets de l'Institution parisienne et s'étant ainsi familiarisé à leur langage naturel ${ }^{7}$ - est le premier professeur entendant à utiliser la langue des signes, et non le français signé, dans l'enseignement des élèves sourds ${ }^{8}$. Il invente une écriture fondée sur l'étude des différents paramètres des signes (Mimographie, 1825). Avec 135 années d'avance, il anticipe, ainsi, sur les analyses 
structurales que le linguiste William Stokoe appliquera dans les années 1960 à la langue des sourds américains ${ }^{9}$. S'intéressant à la description d'un niveau phonologique des signes, William Stokoe (1919-2000) met en évidence la double articulation dans l'American Sign Language, parlant de kinèmes pour les morphèmes gestuels et de chérèmes pour les phonèmes gestuels et relève trois paramètres manuels majeurs : la configuration, l'emplacement et le mouvement.

Où en est la recherche aujourd'hui ? Sans prétendre à l'exhaustivité, ce numéro de la revue TIPA est né du souhait d'établir un état des lieux de cette recherche menée par plusieurs disciplines ${ }^{10}$. Trois types de travaux y sont regroupés : le premier relève de l'ethnologie et de l'histoire des langues des signes; le second traite de la linguistique des langues des signes et le troisième porte sur la traduction, les correspondances morphologiques, la didactique et la parentalité.

Dans une première partie, Yves Delaporte, ethnologue, spécialiste de la langue des signes, présente une analyse étymologique du signe EFFORT au croisement d'une tendance évolutive et d'un champ morphosémantique (article 1) et Aude de Saint Loup, historienne, montre la complexité du questionnement qui ne cesse entre langue des signes et oral, à travers l'histoire des études des langues des signes et de l'éducation des sourds (article 2).

6 Les linguistes de l'Université Paris Lumières-Université Paris 8 - Brigitte Garcia, MarieAnne Sallandre, Marie-Thérèse L'Huillier et Hatice Aksen - exposent les principales stratégies d'expression de la référence impersonnelle humaine en langue des signes française (article 3). Le travail d'Aurélie Collomb, Annelies Braffort et Sylvain Kahane, spécialistes de la modélisation et du traitement automatique des langues, porte sur l'analyse linguistique de certains proformes à partir de l'annotation d'un corpus de langue des signes française, plus particulièrement aux proformes qui servent à introduire un référent du discours (article 4). Quant à l'étude de Myriam Charpentier, celle-ci tente d'identifier le fonctionnement syntaxique de la langue des signes française, dont l'argument sujet (article 5). Toutefois, le corpus des données signées est surtout décrit selon une manière de faire du sens à partir d'images plus que de procédés langagiers. Annie Risler suggère un changement de regard sur la langue des signes (article 6). Nicolas Tournadre et Mélanie Hamm montrent qu'au-delà de ses spécificités structurelles et fonctionnelles, la langue des signes française partage des caractéristiques typologiques que l'on retrouve dans certaines familles de langues vocales ainsi que dans des langues écrites de type logographiques (article 7).

Dans la troisième partie de ce numéro, Pénélope Houwenaghel et Annie Risler interrogent la poésie signée, le travail des traducteurs et la performance scénique (article 8). Aliéner Bouchaud et Hélène Giraudo proposent, quant à elles, d'étudier la nature des relations morphologiques lexicales entre la langue des signes française et le français écrit, au travers d'une étude linguistique comparative, questionnant ainsi la problématique de l'apprentissage de la lecture par des enfants sourds (article 9). Véronique Geffroy et Élise Leroy interrogent, pour leur part, la pertinence de créer une didactique de la langue des signes française (article 10). Les chercheurs canadiens, Charles Gaucher et Sarah Kirsch, présentent une étude qualitative menée auprès de 52 parents d'enfant ayant une surdité et s'intéressent aux défis linguistiques et identitaires perçus par ces parents (article 11).

Ce $34^{\mathrm{e}}$ numéro de TIPA est dédié à Yves Delaporte, ancien directeur de recherche au $\mathrm{CNRS}^{11}$. Questionneur infatigable, passionné et érudit, il signe une centaine d'ouvrages 
et d'articles consacrés à la langue des signes. C'est sans doute l'auteur scientifique le plus lu et relu par les sourds et les signeurs. Véritable Champollion de la langue des signes, il se voue, actuellement, à l'étymologie des signes et au recensement des variations régionales ${ }^{12}$.

9 Les 11 articles, rédigés par 20 chercheurs, dont deux sourds signeurs de naissance ${ }^{13}$, montrent la diversité et l'originalité des études menées autour de la langue des signes ${ }^{14}$. Elles sont au croisement de nombreuses disciplines, mais rarement réunies dans un même numéro, de plus francophone ${ }^{15}$. Puissent ces éclairages donner la force d'engager un combat contre le renoncement et la fatalité. Car la langue des signes est une langue fragile mais résiliente ${ }^{16}$. Toutes les langues appartiennent au patrimoine immatériel de l'humanité (Unesco, 2003). La langue des signes possède pourtant une spécificité unique : ce n'est pas une langue orale. Pas de voix ni d'oreilles, mais des yeux et des mains. Elle témoigne de l'intelligence humaine et prouve que le besoin fondamental de communiquer est plus fort que certains écueils. La langue des signes peut servir dans toutes les circonstances où la voix ne passe pas. Sa singularité se manifeste, également, dans le type de relation qu'elle institue: on ne peut utiliser la langue des signes sans être attentif à l'autre ou en lui tournant le dos. Ceci explique son irremplaçable intérêt et sans doute aussi son attrait. La langue des signes, c'est comme ça.

\section{BIBLIOGRAPHIE}

Aparicio, M. (2007) Étude neuropsychologique et neuroanatomofonctionelle du traitement phonologique chez le lecteur sourd pré-lingual, Thèse de doctorat ès neuropsychologie, Université Louis Pasteur de Strasbourg, $173 \mathrm{p}$.

Bamberg, A. (1998) Passions autour des signes et confession du sourd. Enquête à partir de manuels de morale en tradition catholique, Praxis juridique et religion (PJR), 15, p. 97-155.

Bébian, A. (1817) Essai sur les sourds-muets et sur le langage naturel ou Introduction à une classification naturelle des idées avec leurs signes propres, Paris : J. G. Dentu, 150 p.

Bébian, A. (1825) Mimographie ou Essai d'écriture mimique, propre à régulariser le langage des sourdsmuets, Paris : L. Colas, 42 p.

Benvenuto, A. (2009), Qu'est-ce qu'un sourd ? De la figure au sujet philosophique, Thèse de doctorat ès philosophie, Université de Paris 8, 338 p.

Bernard, Y. (2014) L'esprit des sourds : les signes de l'Antiquité au XIXème siècle [DVD], Les Essarts-leRoi : Éditions du Fox, 1350 p.

Bertin, F. (2010) Les Sourds. Une minorité invisible, Paris : Éditions Autrement (Mutations), 182 p.

Bertin, F. (2015) Auguste Bébian et les Sourds : le chemin de l'émancipation, Thèse de doctorat ès histoire, Université de Poitiers, 2 vol.

Brétéché, S. (2015) L'incarnation musicale. L'expérience musicale sourde, Thèse de doctorat ès musicologie, Aix-Marseille Université, 747 p.

TIPA. Travaux interdisciplinaires sur la parole et le langage, 34 | 2018 
Courtin, C. (1998) Surdité, langue des signes et développement cognitif, Thèse de doctorat ès psychologie cognitive, Université Paris Descartes, 299 p.

Cuxac, C. (1983) Le langage des sourds, Paris : Payot, 206 p.

Cuxac, C. (1993) Iconicité des langues des signes, Faits de Langues, 1, p. 74-56.

Cuxac, C. (2000) La langue des signes française : les voies de l'iconicité, Faits de Langues,15-16, Paris-Gap : Ophrys, $391 \mathrm{p}$.

Cuxac, C. \& E. Antinoro-Pizzuto (2010) Émergence, normes et variation en langue des signes : vers une redéfinition conceptuelle, Langage \& société, 131, Sourds et langues des signes : norme et variations, p. 37-53.

Dagron, J. (1999) Sourds et soignants, deux mondes, une médecine, Paris : Press. 173 p.

Delaporte, Y. (2002) Les sourds, c'est comme ça, Paris : Maison des sciences de l'homme, 375 p.

Delaporte, Y. (2017) Communication personnelle [courrier électronique].

Dictionnaire alphabétique et analogique de la langue française (2001). Le Petit Robert [CD-ROM].

Drion, B. (2018) Préface, in Belissen, P., Angèle, P., Chemoun, N., Puncola, O., Sangla, J., Vergès, J. \& P. Gache, Paroles de sourds : à la découverte d'une autre culture, Paris : La Découverte, 270 p.

Dumont, A. (2008) Orthophonie et surdité : communiquer, comprendre, parler, Issy-les-Moulineaux : Elsevier Masson, $241 \mathrm{p}$.

Flory, D. (2005) 100 exercices : une solution multimédia bilingue (langue des signes-français). Enseigner et apprendre en LSF : vers une éducation bilingue [Dossier], La Nouvelle revue de l'AIS : adaptation et intégration scolaires, hors-série, p. 109-118.

Gaucher, C. (2009) L'altérité des Sourds : deux lieux communs pour interroger la liminalité des sociétés individualistes, Monde Commun, 1, p. 1-14.

Hervé, B. (2014) Les impasses actuelles du pédagogique et les enjeux de l'accessibilité face au défi éthique de l'inclusion sociale, Thèse de doctorat ès Sciences de l'Éducation et psychanalyse, Université Paul Valéry Montpellier 3.

Karacostas, A. (1999) Editorial, Surdités, Revue internationale, 1.

L'Épée, C.-M. de (1776) Institution des sourds-muets par la voie des signes méthodiques, Paris : Nyon l'aîné, 2 parties, 228 p., 132 p.

Lepot-Froment, C. \& N. Clerebaut (1996) L'enfant sourd. Communication et langage, Bruxelles : De Boeck Université, $672 \mathrm{p}$.

Leybaert, J. (2011) (dir.) La Langue française parlée complété, Marseille : Solal, 270 p.

Mottez, B. (1977) À s'obstiner contre les déficiences, on augmente souvent le handicap. L'exemple des sourds, Sociologie et Société, 1, p. 20-32.

Oléron, P. (1972) Langage et développement mental, Bruxelles : Charles Dessart, 299 p.

Pelletier, A. \& Y. Delaporte (2002) Moi, Armand, né sourd et muet. Au nom de sa science, la langue des signes sacrifiée, Paris : Plon (Terre humaine), 462 p.

Poizat, M. (1996) La Voix sourde. La société face à la surdité, Paris : Métailié, 291 p.

Sabria, R. (2006) Sociolinguistique de la langue des signes française, Glottopol, 7, p. 6-31.

Virole, B. (1996) Psychologie de la surdité, Bruxelles : De Boeck Université (Questions de personne), $459 \mathrm{p}$.

TIPA. Travaux interdisciplinaires sur la parole et le langage, 34 | 2018 


\section{Autre référence}

« Yves Delaporte, chasseur de signes », dans l'émission L'œil et la main, France 5, décembre 2017, https://www.france.tv/france-5/l-oeil-et-la-main/345105-yves-delaporte-chasseur-designes.html.

\section{NOTES}

1. Voir Bernard, $2014: 59$.

2. Bébian, 1817.

3. Bébian, 1825.

4. Cuxac, 1983.

5. Le Petit Robert, 2001.

6. Delaporte, in Pelletier \& Delaporte, 2002 : 263.

7. Bernard, $2014: 341$.

8. « C'est à Saint-Jacques, le premier professeur tout court à faire cela ! Massieu et Clerc faisaient du français signé » (communication personnelle d'Y. Delaporte, avril 2018).

9. Delaporte, in Pelletier \& Delaporte, $2002: 283$.

10. En linguistique (Cuxac, 1983, 1993, 2000), en sociolinguistique (Sabria, 2006), en psycholinguistique (Leybaert, 2011), en psychologie (Oléron, 1972 ; Lepot-Froment \& Clerebaut, 1996 ; Virole, 1996 ; Courtin, 1998), en neurolinguistique (Aparicio, 2007), en pédagogie (Flory, 2005), en orthophonie (Dumont, 2008), en sciences de l'éducation (Hervé, 2014), en anthropologie (Gaucher, 2009), en sociologie (Mottez, 1977), en ethnologie (Delaporte, 2002), en histoire (Bertin, 2010, 2015), en psychanalyse (Poizat, 1996), en médecine (Karacostas, 1999 ; Dagron, 1999 ; Drion, 2018), en philosophie (Benvenuto, 2009), en lettres (Bernard, 1941), en théologie (Bamberg, 1998), en musicologie (Brétéché, 2015), pour ne citer que quelques auteurs et travaux francophones, et sans compter les écrits des sourds.

11. Yves Delaporte n'est pas un linguiste, mais son parcours est celui d'un chercheur qui tente de comprendre cette source du langage qu'est la langue des signes.

12. Voir "Yves Delaporte, chasseur de signes ", dans l'émission L'œil et la main, décembre 2017.

13. Tous ces chercheurs travaillent ou ont travaillé en étroite collaboration avec la communauté sourde et malentendante.

14. Nous tenons à remercier les auteurs pour leur contribution, ainsi que le comité scientifique et l'équipe TIPA, en particulier Martine Faraco, rédactrice en chef, pour son soutien et ses précieuses relectures.

15. Pour ces chercheurs, il semble important que leurs travaux soient connus du public des lecteurs francophones: le plus grand nombre d'entre eux publient en anglais, ce qui exclut beaucoup de lecteurs sourds. Certains articles de ce numéro seront résumés en langue des signes, sur le site de TIPA.

16. Ainsi que l'écrit Christian Cuxac (2010: 41) : «Les LS montrent, à côté de leur fragilité potentielle, une résilience extraordinaire. Quelle langue vocale sans localisation géographique précise, sans écriture, avec une fragmentation si dramatique de ses locuteurs, avec un nombre si faible de locuteurs natifs, aurait pu être transmise aussi longtemps? Quelle langue vocale aurait pu résister et se développer principalement par l'intermédiaire de locuteurs "non natifs" et aussi diversifiés?». 


\section{AUTEUR}

\section{MÉLANIE HAMM}

Aix-Marseille Université, CNRS, LPL, Aix-en-Provence, France

Melanie.hamm@univ-amu.fr 